\title{
Study on the Rainfall Interpolation Algorithm of Distributed Hydrological Model Based on RS
}

\author{
Xiaoxia Yang, Yong Liang, and Song Jia \\ School of Information Science and Engineering, \\ Shandong Agricultural University, \\ Taian, Shandong Province, P. R. China, 271018 \\ yangxx@sdau.edu.com
}

\begin{abstract}
Distributed hydrological model can be divided into two parts, as runoff and the convergence. Runoff calculation is the basis of distributed hydrological model, its results will determine the accuracy of the simulation results directly. Rainfall is an important input of runoff calculation, thus its accuracy has special significance to runoff calculation. For most small watershed, now mainly rely on rainfall stations to obtain rainfall information. In this case, the most effective approach is base on space correlativity principle, use the interpolation algorithm to obtain distributed rainfall data. Take XueYe reservoir 2000-2008 year of reality measures data as an example, we compared the apply condition of several kinds algorithm such as co-kriging interpolation, kriging interpolation, reverse-weighted interpolation, and so on, prove that co-kriging interpolation is most fit XueYe reservoir .
\end{abstract}

Keywords: Distributed hydrological model, Association Kriging interpolation, Kriging interpolation, Reverse-weighted interpolation.

\section{Introduction}

Distributed hydrological model has been a hot research field for nearly 20 years [1], what can be divided into two parts, as runoff and the convergence. Runoff calculation is the basis of distributed hydrological model, its results will determine the accuracy of the simulation results directly. Rainfall is an important input of runoff calculation, its spatial distribution characteristics is the main control factors of runoff and a series of other hydrological problems. The effective method to get accurate rainfall distribution characteristics is to set density rainfall station observation network [2], but now most small watershed has limited quantity of rainfall station and the distribution of rainfall stations are always not reasonable, so the data from these stations often cannot meet the requirements. Therefore, the spatial interpolation method according to the acquired data become a research hotspot. There are several interpolation methods [3] [4] [5] [6] [7], different method has different results, and no one is common interpolation method for optimum interpolation method [8] [9].Taking XueYe reservoir 2000-2008 year of reality measured data as an example the paper contrast cokriging interpolation, kriging interpolation, reverse-weighted interpolation and thiessen polygon interpolation, then analyses the interpolation method fit XueYe reservoir. 


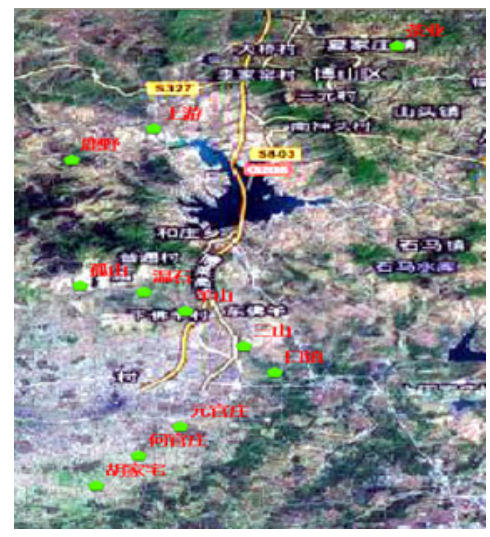

Fig. 1. 11 rainfall stations's scattergram of XueYe reservoir

XueYe reservoir is one of the large reservoir in ShanDong province, founded in 1959.XueYe reservoir has a total capacity of 2.21 billion cubic meters, water area of 1.8 million acres. It located in $117.54 \sim 117.64$ degrees east longitude and latitude between 36.39 36.46 degrees, belong to temperate continental monsoon climate, the four seasons, the annual average temperature is between 11.0- 13.0 degrees Celsius, precipitation $760.9 \mathrm{~mm}$. Figure 1 is about 11 rainfall stations's scattergram of XueYe reservoir.

\section{Rainfall Space Interpolation Method}

First, compare the relationship between the XueYe 2000-2008 reality measures rainfall data and longitude, latitude, elevation, found that rainfall data with latitude, longitude linear correlation is not obvious, the correlation coefficient $R^{2}$ are -0.52 and -0.47. But the rainfall data with elevation is obvious, $\mathrm{R}^{2}=0.6021$.

\subsection{Thiessen Polygon Interpolation}

Thiessen polygon interpolation is one of the most simple partial interpolation algorithm, put forward by the Dutch climatologists A.H. Thiessen. Its main idea is adjoin all rainfall stations by triangle, for each side of the triangle do vertical bisectrix, each station around several vertical bisectrix form a polygon, and the polygon contain only one station, the polygon called Thiessen polygon. Thus we can express the data of all the points in the polygon by the station's reality measures data.

Thiessen polygon interpolation is simple. In the case that there are enough rainfall stations, Thiessen polygon interpolation can be good at approximating the actual value. It's shortcoming is the mutation on the boundary. It can't fit the space characteristics that rainfall is gradually changing. Meanwhile Thiessen polygon interpolation neglect the influence of elevation, not suitable for data interpolation of XueYe reservoir. 


\subsection{Reverse-Weighted Interpolation}

Reverse-weighted interpolation is one of the space geometric interpolation method. When we use the sampling points estimate the estimation point, often farther to the estimate point smaller the influence is, in other words farther sampling point has smaller weight. Thus, the value of eatimate point $\mathrm{Z}\left(\mathrm{x}_{0}\right)$ can be fitting by all the surrounding point's linear weighted:

$$
Z\left(x_{0}\right)=\sum_{i=1}^{n} \frac{1}{(D i)^{P}} Z\left(x_{i}\right) / \sum_{i=1}^{n} \frac{1}{(D i)^{P}}
$$

$D i$ is the distance from the $\mathrm{i}$ sampling point to eatimate point, $Z(x i)$ is the value of $\mathrm{i}$ sampling point, index $p$ is the power of distance, used to control the weight's change speed with distance. Different value of $p$ will influence the result of interpolation, the bigger the value of $p$ the smaller weight of the far distance point. $p$ can values $1,2,3$. When $p=2$ is called the inverse distance square interpolation method. In this paper the value of $p$ is 2.The strong point of reverse-weighted interpolation is it can adjust the result of spatial interpolation by weight, but the shortcoming is it also neglect the influence of elevation.

\subsection{Kriging Interpolation}

Kriging interpolation is regarded as one of the main method of geological statistics, is raised by South Africa scientist D.G. Krige in 1951 and named by his name. Kriging interpolation fully absorb the idea of space statistics, think any space of continuous change attributes is very irregular, can't imitation by simple smooth mathematical functions, but can described by random surface function. From the angle of interpolation Kriging interpolation is one of the method that is linear interpolation optimal, unbiased estimation for directional distribution data. Kriging interpolation is defined as:

$$
\mathrm{Z}\left(\mathrm{x}_{0}\right)=\sum_{i=1}^{n} \lambda_{i} Z\left(x_{i}\right)
$$

$Z(x i)$ is the observed value of i point, $Z\left(x_{0}\right)$ is the value of estimation point, $\lambda_{i}$ is the power, and we have

$$
\sum_{i=1}^{n} \lambda_{i}=1
$$

The linchpin of kriging interpolation is to fix the value of power $\lambda_{i}$,it should make the value of $Z\left(x_{0}\right)$ unbiased estimation, that is less than any variance of linear combination observations of the variance. In the ordinary use half variance as a basis to fix the value of the power. So at the first we should fix the model of half variance, the common model of half variance are nugget, spherical surface, index, Gauss, power and linear model. In the paper we adopt spherical model. 


\subsection{Co-kriging Interpolation}

The theory of co-kriging interpolation is similar with kriging interpolation. Co-kriging interpolation optimal the estimation through the way that consider moer than one variables and the relation between the variables. Use co-kriging interpolation,we can use the correlation between the variables, estimate the value of one or more variables, improve the accuracy and rationality of estimation. Co-kriging interpolation introduce the crossover-mutation function, that is the function that the correlation of two different variables change with the distance. The crossover-mutation function is defined as :

$$
\mathrm{r}_{\mathrm{ij}}(\mathrm{h})=1 / 2 * \mathrm{E}\left\{\left[\mathrm{Z}_{\mathrm{i}}(\mathrm{x}+\mathrm{h})-\mathrm{Z}_{\mathrm{i}}(\mathrm{x})\right]\left[\mathrm{Z}_{\mathrm{ji}}(\mathrm{x}+\mathrm{h})-\mathrm{Zj}(\mathrm{x})\right]\right\}
$$

Within XueYe reservoir the elevation is knowed ererywhere and stable,we introduce the elevation as a factor into co-kriging interpolation.

\section{Analysis the Result of Rainfall Interpolation}

\subsection{Method of Calibration}

Adopt cross validation methods to analysis the result of interpolation: randomly extract 2 rainfall stations as docimastic station among all the 11 rainfall stations of XueYe reservoir, remove the data of the docimastic station, use data of the rest 9 rainfall stations to estimate the value of docimastic staion, and compute the error. Use mean absolute error(MAE) and root mean squared interpolation error(RMSIE) as the evaluation criterion to evaluate the result of interpolation. MAE is used to evaluate the error range, RMSIE can reflect valuations sensitivity and maximum effect of interpolation.

$$
\begin{aligned}
M A E & =\sum_{i=1}^{n}\left|Z^{\prime}\left(x_{i}\right)-Z\left(x_{i}\right)\right| / n . \\
R M S I E & =\sqrt{\sum_{i=1}^{n}\left[Z^{\prime}\left(x_{i}\right)-Z\left(x_{i}\right)\right]^{2} / n} .
\end{aligned}
$$

Table 1. The result of reverse-weighted interpolation

\begin{tabular}{|c|c|c|c|c|c|c|c|c|c|c|c|c|c|}
\hline \multicolumn{2}{|c|}{ Month } & 1 & 2 & 3 & 4 & 5 & 6 & 7 & 8 & 9 & 10 & 11 & 12 \\
\hline II & $\begin{array}{c}\text { Original } \\
\text { data }\end{array}$ & 0.42 & 0.78 & 1.12 & 1.12 & 2.99 & 6.53 & 13.01 & 8.53 & 5.69 & 2.01 & 0.91 & 0.56 \\
\cline { 2 - 13 } & $\begin{array}{c}\text { Square root } \\
\text { transformation }\end{array}$ & 0.41 & 0.8 & 1.1 & 1.11 & 2.92 & 6.49 & 12.97 & 8.51 & 5.71 & 1.93 & 0.86 & 0.51 \\
\hline $\begin{array}{l}\text { R } \\
\text { II } \\
\text { S }\end{array}$ & $\begin{array}{c}\text { Original } \\
\text { data }\end{array}$ & 0.57 & 0.93 & 1.66 & 1.77 & 3.92 & 7.74 & 16.51 & 10.77 & 6.75 & 2.87 & 1.37 & 0.75 \\
\hline $\begin{array}{c}\text { I } \\
\text { E }\end{array}$ & $\begin{array}{c}\text { square root } \\
\text { transformation }\end{array}$ & 0.56 & 0.96 & 1.63 & 1.73 & 3.87 & 7.67 & 16.44 & 10.77 & 6.78 & 2.81 & 1.32 & 0.72 \\
\hline
\end{tabular}


Table 2. The result of kriging interpolation

\begin{tabular}{|c|c|c|c|c|c|c|c|c|c|c|c|c|c|}
\hline \multicolumn{2}{|r|}{ Month } & 1 & 2 & 3 & 4 & 5 & 6 & 7 & 8 & 9 & 10 & 11 & 12 \\
\hline \multirow{2}{*}{$\begin{array}{l}\text { M } \\
\mathrm{A} \\
\mathrm{E}\end{array}$} & Original data & 0.37 & 0.71 & 0.95 & 1. 12 & 2. 97 & 6.4 & 12.36 & 8.34 & 5.11 & 1. 94 & 0.87 & 0.49 \\
\hline & $\begin{array}{c}\text { square root } \\
\text { transformation }\end{array}$ & 0.34 & 0.53 & 1.01 & 1.05 & 2. 77 & 6. 14 & 12.2 & 8.46 & 5. 31 & 1.81 & 0.8 & 0.45 \\
\hline \multirow{2}{*}{$\begin{array}{c}\text { R } \\
\text { II } \\
\text { S } \\
\text { I } \\
\text { B }\end{array}$} & Original data & 0.52 & 0.97 & 1. 46 & 1.7 & 3. 85 & 7. 56 & 15.77 & 10.59 & 6. 13 & 2. 77 & 1. 34 & 0.76 \\
\hline & $\begin{array}{l}\text { square root } \\
\text { transformation }\end{array}$ & 0.49 & 0.79 & 1.54 & 1.6 & 3. 67 & 7. 32 & 15.42 & 10.72 & 6.34 & 2. 67 & 1.24 & 0.71 \\
\hline
\end{tabular}

Table 3. The result of co-kriging interpolation

\begin{tabular}{|c|c|c|c|c|c|c|c|c|c|c|c|c|c|}
\hline \multicolumn{2}{|r|}{ 月份 } & 1 & 2 & 3 & 4 & 5 & 6 & 7 & 8 & 9 & 10 & 11 & 12 \\
\hline II & Original data & 0.34 & 0.62 & 0.93 & 1.09 & 2. 96 & 6. 33 & 12. 16 & 8.33 & 5.06 & 1. 92 & 0.86 & 0.47 \\
\hline A & $\begin{array}{c}\text { square root } \\
\text { transformation }\end{array}$ & 0.32 & 0.57 & 0.91 & 1.04 & 2. 73 & 6. 37 & 12. 27 & 8.21 & 4. 49 & 1. 78 & 0.84 & 0.42 \\
\hline $\mathrm{R}$ & Original data & 0.49 & 0.88 & 1. 44 & 1.62 & 3.84 & 7. 49 & 15. 36 & 10.57 & 6. 08 & 2. 73 & 1.35 & 0.74 \\
\hline $\begin{array}{l}\text { S } \\
\text { I } \\
\text { E }\end{array}$ & $\begin{array}{l}\text { square root } \\
\text { transformation }\end{array}$ & 0.48 & 0.81 & 1.41 & 1.58 & 3. 61 & 7.54 & 15.49 & 10.48 & 5.49 & 2. 62 & 1.3 & 0.69 \\
\hline
\end{tabular}

$Z_{i}$ is the actual observed value of the i point, $Z^{\prime}{ }_{i}$ is the estimation value of it, $n$ is the amount of docimastic station. Prepeocess the data will make the data tends to normal distribution and improve the accuracy of estimation. In this paper we adopt square root transformation to improve the accuracy of estimation.

\subsection{Analysis the Result of Interpolation}

Paper use square root transformation, adopt reverse-weighted interpolation, kriging interpolation, co-kriging interpolation, get 6 results of interpolation. Compare the 6 results, find the best interpolation fits to XueYe reservoir.

Analysised years of data we find that: most month have better interpolation accuracy after square root transformation. Compare with original data co-kriging interpolation after square root transformation has better interpolation accuracy in month $1,2,3,4,5,8,9,10,11,12$.Compare with original data kriging interpolation after square root transformation has better interpolation accuracy in month 1,2,4,5,6,7,10,11,12. Compare with original data reverse-weighted interpolation after square root interpolatio has better interpolation accuracy in month 1,3,4,5,6,7,8,10,11,12.

Compare with the other two interpolations after square root transformation cokriging interpolation has better interpolation accuracy in month 1, 3, 4, 5, 8, 9, 10, 12. Original co-kriging interpolation has better interpolation accuracy in month 7 . After square root transformation kriging interpolation has better interpolation accuracy in month 2,6,11.

The MAE of co-kriging interpolation after square root transformation is 3.33 , RMSIE is 4.29; The MAE of original data co-kriging interpolation is 3.42,RMSIE is 4.38; The MAE of kriging interpolation after square root transformation is 3.41 , RMSIE is 4.38; The MAE of original data kriging interpolation is 3.47, RMSIE is 
4.45; The MAE of reverse-weighted interpolation after square root transformation is 3.53, RMSIE is 4.61; The MAE of original data reverse-weighted interpolation is 3.64, RMSIE is 4.63 .

Through the analysis of data we find that, co-kriging interpolation after square root transformation has a higher precision than the other interpolation, is more suitable for the actual situation XueYe reservoir.

\section{Conclusion}

Analysised years of data we find that:the rainfall of XueYe has relatively close relationship with elevation, so the interpolation irrelevant with elevation is not fit to this area. Because after square root transformation the data will tends to normal distribution, so before interpolation we adopt square root transformation, experimental data shows that after square root transformation most month have better result. After analysis several common interpolation, we get the following conclusion: co-kriging interpolation after square root transformation is fit to XueYe reservoir.

\section{Acknowledgements}

This study has been funded by Office of ShanDong Government Flood Control and Drought Relief Headquarters. The project name is the research and development of the system of optimal scheduling about flood resource. It is supported by Shandong Agricultural University. Sincerely thanks are also due to XueYe reservoir for providing the data for this study.

\section{References}

1. Goovaerts, P.G.: Approaches for incorporating elecation into the spatital interpolation of rainfall. Journal of Hydrology (228), 1113-1291 (2000)

2. Band, C.: Forest ecosystem process at the warter scale:Basis for distributed simulation. Ecol. (56), 171-196 (1991)

3. Christopher, D., Waync, P.G., George, H.T., Gregory, L.J., Phillip, P.: A knowledge-based approach to the statistical mapping of climate. Climate Research (22), 99-113 (2002)

4. Dabid, T.P., Daniel, W.M., Ian, A.: A comparison of two statistical methods for spatital interpolation of Canadian monthly mean climata data. Agriculutural and Forest Meteotology (101), 81-94 (2000)

5. Dirks, K.N., Stow, C.D.: Highresolution studies of rainfall on Norfolk Island, Part II:interpolation of rainfall data. J. Hudrol. (208), 187-193 (1998)

6. Bartier, P.M., Keller, C.P.: Multivate interpolation to incorporate thernatic surface data using inverse distance weighting. Computer \& Geoscience (22), 795-799 (1996)

7. Huiyi, Z., Shaofeng, J.: Uncertainly in the spatial interpolation of rainfall data. Progress in Geography (23), 34-41 (2004)

8. Houghton, J., Meira, L.G., Callander, B.A.: Change 1995: the Science of Climate Change. Journal of Hydrology (1996) 